\title{
Rapid detection of Mycobacterium tuberculosis and Rifampicin Resistance in extra pulmonary samples using Gene Xpert MTB/RIF assay
}

\author{
Ahmad Naeem Sajed ${ }^{1}$, Sana khan ${ }^{2}$, Aneeqa Shamshad Butt ${ }^{2}$, Abid Srwar ${ }^{3}$, Dr. \\ Raheela Akhtar ${ }^{4}$, Dr. Imran Ahmad ${ }^{4}$, Muhammad Naeem Mukhtar ${ }^{1}$, Dr. Sadaf \\ Imran $^{4}$, Dr. Hamid Akbar ${ }^{4}$, Shan $\mathrm{Ali}^{3}$, Muhammad Usman ${ }^{3}$ \\ ${ }^{1}$ Emergency Services Academy Rescue 1122 Lahore-Pakistan \\ ${ }^{2}$ Pathology Department Allama Iqbal Medical College Lahore-Pakistan \\ ${ }^{3}$ Institute of Molecular Biology and Biotechnology the University of Lahore-Pakistan \\ ${ }^{4}$ University of Veterinary and Animal Sciences Lahore-Pakistan
}

\begin{abstract}
Tuberculosis (TB) is a major health problem in Pakistan. The World Health Organization has recently endorsed the Gene Xpert MTB/RIF assay for rapid detection of smear negative and multidrug resistance tuberculosis. A total of 100 extra pulmonary samples were processed, which included 60 pus, 19 pleural fluids, 16 ascetic fluids and 5 CSF. Out of these 37\% patients were Gene Xpert MTB/RIF Assay positive, $17 \%$ were LJ culture positive and $12 \%$ were Zn smear positive. MTB was detected in 31 out of 60 (51.7\%) Pus samples, 3 out of 19 (15.8\%) Pleural fluid samples, 1 out of 16 (6.3\%) Ascitic fluid samples and 2 out of 5 (40.0\%) CSF samples. In this study we found that Gene Xpert MTB/RIF assay is rapid method for diagnosis of extra pulmonary tuberculosis as compared to conventional methods. Because of its simplicity, rapidity and sensitivity, this seems to be a very gorgeous tool for diagnosis of extra pulmonary tuberculosis from clinical samples.
\end{abstract}

Keywords: Extra pulmonary tuberculosis, Mycobacterium tuberculosis, Gene Xpert MTB/RIF assay

\section{Introduction}

Tuberculosis is the commonest infectious disease worldwide caused by Mycobacterium tuberculosis. According to WHO there were 8.6 million new TB cases in 2012 and even 1.3 million TB deaths. ${ }^{1}$ In the underdeveloped countries $95 \%$ of infections occur due to pitiable diagnostic and treatment facilities. It is estimated that approximately 70 million people will die from tuberculosis within next 20 years and it is because of inadequate measures for the TB control ${ }^{2}$.

Tuberculosis is typically divided into two types, pulmonary which is more widespread and extra pulmonary which rivet $15 \%$ cases. Extra pulmonary tuberculosis (EPTB) can involve any organ in the body. Extra pulmonary infections with members of the Mycobacterium tuberculosis complex (MTBC) have high morbidity and mortality because of lack of good diagnostic methods. Diagnosis is often difficult to establish due to low number of bacteria and collection of extra pulmonary samples is not easy. A definitive diagnosis of mycobacterium infection depends on detection of the Mycobacterium Tuberculosis in extra pulmonary samples ${ }^{3}$.

The frequently atypical clinical presentation simulating other inflammatory and neo-plastic conditions results in a delay or deprivation of treatment which is the major challenge in the control of EPTB. Radiography provides useful information in the diagnosis of EPTB. Therefore, a high index of suspicion is necessary to make an early diagnosis and quite often, more than one procedure is necessary for the confirmation of the diagnosis. In lower-income countries, the lack of diagnostic infrastructure substantially aggravates the problem. Now attention has been devoted to latest nucleic acid amplification diagnostic systems due to their speed, sensitivity and specificity. ${ }^{4}$

The World Health organization has recommended the use of gene Xpert for rapid detection of MTB in extra pulmonary samples. The gene Xpert MTB/RIF assay detects DNA sequences specific for Mycobacterium tuberculosis and Rifampicin resistance by polymerase chain reaction ${ }^{5}$. It is based on the Cepheid Gene Xpert system, a platform for rapid and simple-to-use. The Xpert MTB/RIF purifies and concentrates the Mycobacterium tuberculosis bacilli from extra pulmonary samples, isolates genomic material and amplifies the genomic DNA by PCR. Results are obtained in 90 minutes from extra pulmonary samples, with minimal biohazards. Further minimal technical training is required to operate the instrument. These features make it an important tool for extra pulmonary samples ${ }^{6}$. 


\section{Material And Methods}

This study was conducted in Microbiology Laboratory, Pathology Department, Allama Iqbal Medical College Lahore-Pakistan.

\section{Sample collection}

A total of 100 clinically suspected cases of extra pulmonary tuberculosis were selected from Jinnah Hospital, Lahore-Pakistan. Extra pulmonary samples (Pus, CSF, Ascitic fluid, Pleural fluid) were collected in plain universal $30 \mathrm{ml}$ clear plastic container with white cap. Samples were divided into three portions, one for AFB smear, second for LJ culture medium and third for Gene Xpert MTB/RIF assay.

\section{Culture medium}

Specimen was processed by the standard $N$-acetyl-L-cysteine and sodium hydroxide (NALC/NaOH) method with a final $\mathrm{NaOH}$ concentration of $1 \%$. After the centrifugation step, Lowenstein-Jensen (LJ) slant was inoculated with $0.1 \mathrm{ml}$ suspension and incubated at $37^{\circ} \mathrm{C}^{7}$.

\section{AFB smears}

After processing the specimens, smears were prepared from all samples other than urine and were examined at the German National Reference Laboratory for Mycobacteria (NRC) intended for the presence of AFB. All smears were stained by the Kinyoun method and examined with a light microscope ${ }^{8}$.

\section{Xpert procedure}

Sample reagent was added in a 2:1 ratio to $0.5 \mathrm{ml}$ of decontaminated specimen. The closed tube was manually agitated two times during a 15-min incubation period at room temperature before $2 \mathrm{ml}$ of the inactivated sample; reagent-sample mixture was transferred to the Xpert test cartridge. Cartridges were inserted into the GeneXpert device and the automatically generated results were read after $90 \mathrm{~min}^{9}$.

\section{Result And Discussion}

Mycobacterium tuberculosis is a serious public health problem worldwide. Conventional laboratory techniques like direct microscopy is less sensitive and culture are time consuming for the diagnosis of tuberculosis. Therefore it is needed to develop new techniques for rapid identification of the Mycobacterium tuberculosis for TB especially for extra pulmonary samples. Recently attention has been devoted to latest nucleic acid amplification diagnostic systems due to their speed and accuracy. The World Health organization has recommended the use of gene Xpert for rapid detection of MTB in extra pulmonary samples ${ }^{9}$. Entirety, 100 extra pulmonary samples (60 pus, 19 pleural fluids, 16 ascitic fluids and 5 CSF samples) were processed.

Table 1 Breakdown of samples in extra pulmonary Tuberculosis

\begin{tabular}{|c|c|c|}
\hline Specimen type & Frequency & Percent \\
\hline Pus & 60 & 60.0 \\
\hline Pleural fluid & 19 & 19.0 \\
\hline Ascetic fluid & 16 & 16.0 \\
\hline CSF & 5 & 5.0 \\
\hline Total & 100 & 100.0 \\
\hline
\end{tabular}

The result of a study revealed a maximum positivity rate by Gene Xpert which indicated that it is a most sensitive technique as compared to conventional methods. Raquel Moure et al, in 2012 conducted a study; in this research, out of 108 smear-negative extra pulmonary samples $63(58.3 \%)$ were positive with the Xpert MTB/RIF assay (GX) for Mycobacterium tuberculosis ${ }^{10}$. In a similar study by Vadwai in 2011, the sensitivity of the Xpert assay was $81 \%$ (228/283 specimens), $64 \%$ for smear-negative cases and $96 \%$ for smear-positive cases), with a specificity of $99.6 \%$. The higher detection rate in above mention studies was due to the fact that they included diagnosed cases of TB while our study was performed on TB suspects ${ }^{11}$. Doris Hillemann in 2011 also compared Gene Xpert MTB/RIF (Xpert) assay system with conventional liquid and solid culture methods. Entirety 521 specimens ( 91 urine, 30 gastric aspirate, 245 tissue, 113 pleural fluid, 19 cerebrospinal fluid and 23 stool specimens) were submitted. The combined sensitivity and specificity of the Xpert assay were calculated to be $77.3 \%$ and $98.2 \%$, respectively. ${ }^{12}$

In present study, MTB was detected in 31 out of $60(51.7 \%)$ pus samples, 3 out of $19(15.8 \%)$ pleural fluid samples, 1 out of $16(6.3 \%)$ ascitic fluid samples and 2 out of $5(40.0 \%)$ cerebrospinal fluid samples. The study revealed that the Xpert test has true diagnostic potential with good sensitivity for specimens such as pus which is difficult to diagnose by other laboratory techniques. Out of five of cerebrospinal fluid samples two were positive by Gene Xpert which was negative on conventional methods. Our findings supported the use of 
Gene Xpert test in routine for extra pulmonary TB diagnosis especially for pus samples where a very high detection rate was observed as compared to conventional techniques.

Table 2 Result of samples analyzed by Gene Xpert MTB/RIF assays

\begin{tabular}{|c|c|c|c|c|}
\hline \multicolumn{5}{|c|}{ Result of Gene Xpert MTB/RIF Assay } \\
\hline Type of Samples & Positive & Percent & Negative & Percent \\
\hline Pus & 31 & $51.7 \%$ & 29 & $48.3 \%$ \\
\hline Pleural Fluid & 3 & $15.8 \%$ & 16 & $84.2 \%$ \\
\hline Ascetic Fluid & 1 & $6.3 \%$ & 15 & $93.8 \%$ \\
\hline CSF & 2 & $40.0 \%$ & 3 & $60.0 \%$ \\
\hline Total & 37 & $37.0 \%$ & 63 & $63.0 \%$ \\
\hline
\end{tabular}

Out of 100 cases, 17 cases of Gene Xpert MTB/RIF assay positive were LJ culture positive, 20 cases of Gene Xpert MTB/RIF assay positive were LJ culture negative, 63 cases of both Gene Xpert MTB/RIF assay and $\mathrm{Zn}$ smear were negative. Out of 100 cases, 12 cases of Gene Xpert MTB/RIF assay positive were Zn smear positive, 25 cases of Gene xpert MTB/RIF assay positive were Zn smear negative, 63 cases of both Gene Xpert MTB/RIF assay and $\mathrm{Zn}$ smear were negative. It is noted that none of the $\mathrm{Zn}$ smear positive and LJ culture positive samples gave negative results by Gene Xpert. On the other hand all the Zn negative and LJ culture negative samples were also negative on Gene Xpert indicating Xpert MTB/RIF assay is highly sensitive and specific technique.

Table 3 Comparison of Gene Xpert assay with Zn smear and LJ culture results

\begin{tabular}{|c|c|c|c|c|}
\hline Gene Xpert and MTB/RIF Assay & \multicolumn{2}{|c|}{ Diagnosis on Ziehl-Nelsen } & \multicolumn{2}{|c|}{ Diagnosis Lowenstein-Jensen Culture } \\
\hline & Positive & Negative & Positive & Negative \\
\hline Positive Results of Gene Xpert & 12 & 25 & 17 & 20 \\
\hline Negative Results MTB/RIF Assay & 0 & 63 & 0 & 63 \\
\hline Total Count & 12 & 88 & 17 & 83 \\
\hline
\end{tabular}

Stephen D Lawn in 2012 used same technique Xpert MTB/RIF for diagnosis of extra pulmonary Tuberculosis. Out of the total of 268 samples the positivity rate observed for tissue biopsies or fine-needle aspirates (35\%), gastric aspirates (23\%), pus (21\%), urine (6\%), CSF $(5 \%)$ and other body fluids i.e. peritoneal, synovial and pericardial $(4 \%)^{13}$. In the diagnosis of tuberculosis time factor has crucial importance. It is very important to note that detection time of MTB/RIF assay was only 2 hours as compared to LJ Culture which take 3-8 weeks the mean turnaround time for culture positivity was 5 weeks and $\mathrm{Zn}$ smear take 1-24 hours approximately in the present study. We can safely recommend Xpert MTB/RIF assay as a new tool for rapid diagnosis of TB. Iqbal in 2011 attempted a study to determine mean detection time for Mycobacterium tuberculosis was 24 days by LJ culture and less than 1 day by smear examination ${ }^{14}$. Siddiqui in 2013 observed that the mean turnaround time for culture positivity was 23.13 days with LJ medium in their study ${ }^{15}$. Gene Xpert assay is not only helpful for rapid detection of MTB but also determining the patient's MDR status in bringing to an end the spread of MDR-TB and decreasing mortality. In the present study none of the cases showed Rifampicin resistance which indicates that MDR TB is not common in extra pulmonary TB cases in our country.

Table 4 Comparison of time to positivity of Gene Xpert MTB/RIF assay, LJ Culture and Zn smear

\begin{tabular}{|l|l|l|l|}
\hline \multicolumn{1}{|c|}{ Tests } & \multicolumn{1}{|c|}{ Time of positivity } & Mean Time of positivity & Time of negativity \\
\hline Gene Xpert MTB/RIF assay & 2 Hours & 2 Hours & 2 Hours \\
\hline LJ Culture & $3-8$ Weeks & 5 Weeks & 8 Weeks \\
\hline Zn Smear & 1 Hours & 1 Hours & $1-24$ Hours \\
\hline
\end{tabular}

\section{Conclusion}

Gene Xpert MTB/RIF assay is efficient and reliable technique for the rapid diagnosis of extra pulmonary $\mathrm{TB}$, especially in smear negative cases. Its simplicity, sensitivity, speed and automation, make this technique a very attractive tool for diagnosis of Mycobacterium tuberculosis from extra pulmonary samples in MDR cases and smear negative cases of TB suspects.

\section{Acknowledgement}

I am very thankful to Pathology department, Allama Iqbal Medical College Lahore Pakistan.

\section{References}

[1]. WHO global tuberculosis report Geneva. Euro surveillance 2013; 18(43): 20615.

[2]. Giri PK, Khuller GK. Is Intranasal Vaccination a Feasible Solution for Tuberculosis? Expert Rev Vaccines 7, 2008, 1341-1356. 
[3]. Doris Hillemann et al, Rapid Molecular Detection of Extra pulmonary Tuberculosis by the Automated Gene Xpert MTB/RIF System. 4, 2011, 1202-1205.

[4]. Agarwal S P. and Chauhan LS. Tuberculosis control in India. Directorate General of Health Services/Ministry of Health and Family Welfare,New Delhi, India 2005.

[5]. Rie VA, Page Shipp L, Scott L, Sanne I, Stevens W. Expert Review Molecular Diagnosis 10, 2010, $937-946$.

[6]. Boehme CC. "Rapid molecular detection of tuberculosis and rifampin resistance" N Engl J Med 363, $2010,1005-1015$.

[7]. Deutsches Institut für Normung. Medical microbiology. Diagnosis of tuberculosis. Part 3. Detection of mycobacteria by culture methods. Beuth Verlag, Berlin, Germany. 1986, 58943 -3.

[8]. Deutsches Institut für Normung. Medizinische Mikrobiologie. Tuberkulosediagnostik. Teil 32. Mikroskopische Methoden zum Nachweis von Mykobakterien. Beuth Verlag, Berlin, Germany 1995, 58943-32.

[9]. Helb, D., et al. Rapid detection of Mycobacterium tuberculosis and rifampin resistance by use of on-demand, near-patient technology. J. Clin. Microbiol. 48, 2010, 229-237.

[10]. Moure R, Martín R, Alcaide F. Effectiveness of an Integrated Real-Time PCR Method for Detection of the Mycobacterium tuberculosis Complex in Smear-Negative Extra pulmonary Samples in an Area of Low Tuberculosis Prevalence. 50(2), 2012, 513.

[11]. Vadwai V, Boehme C, Nabeta P, Shetty A, Alland D, Rodrigues C. Xpert MTB/RIF, a New Pillar in Diagnosis of Extra pulmonary Tuberculosis? 2011; 49(7), 2540-2545.

[12]. Doris Hillemann, Rapid Molecular Detection of Extra pulmonary Tuberculosis by the Automated GeneXpert MTB/RIF System. 4, 2011, 1202-1205.

[13]. Stephen D, Alimuddin IZ. Diagnosis of Extra pulmonary Tuberculosis Using the Xpert MTB/RIF Assay. 10(6), 2012, 631-635.

[14]. Iqbal S, Ahmed R, Adhami SZ, Mumtaz A. Importance of polymerase chain reaction in diagnosis of pulmonary and extra pulmonary tuberculosis. 23(1), 2011.

[15]. Siddiqui MAM, Anuradha PR, Nagaman K, Vishnu PH. Comparison of conventional diagnostic modalities, BACTEC culture with polymerase chain reaction for diagnosis of extra-pulmonary tuberculosis. J Med Allied Sci, 3(2), 2013, 53-58. 